\title{
CARACTERIZAÇÃo QUÍMICA DE GLUCANAS FÚNGICAS E SUAS APLICAÇÕES BIOTECNOLÓGICAS
}

\author{
Maria de Lourdes Corradi da Silva*, Paula Felippe Martinez, Nataly Lino Izeli, Iara Ribeiro Silva, Ana Flora Dalberto \\ Vasconcelos e Marilsa de Stefani Cardoso
}

Departamento de Física, Química e Biologia, Faculdade de Ciências e Tecnologia, Universidade Estadual Paulista Júlio de Mesquita Filho, CP 467, 19060-900 Presidente Prudente - SP

Rosângela Maria Stelutti, Ellen Cristine Giese e Aneli de Melo Barbosa

Departamento de Bioquímica e Biotecnologia, Centro de Ciências Exatas, Universidade Estadual de Londrina, CP 6001, 86051-990 Londrina - PR

Recebido em 28/9/04; aceito em 27/4/05; publicado na web em 24/8/05

\begin{abstract}
CHEMICAL CHARACTERIZATION AND BIOTECHNOLOGY APPLICATIONS OF FUNGAL GLUCANS. This article gives an overview of exopolysaccharides produced by fungi. The structural characterization of $\beta$-D- $(1 \rightarrow 3)$ and $\beta$-D- $(1 \rightarrow 3,1 \rightarrow 6)$ - glucans is discussed focusing on different chemical and physical procedures. The industrial applications are also considered mainly from the point of view of human health.
\end{abstract}

Keywords: exopolysaccharides; glucans; structural characterization.

\section{INTRODUÇÃO}

Os biopolímeros têm sido objeto de intensa pesquisa, tendo em vista seu elevado potencial de aplicação em diferentes setores ${ }^{1}$. Os polissacarídeos de origem microbiana além de apresentarem propriedades similares ou superiores aos tradicionais (de origem vegetal e algas marinhas) são, muitas vezes, capazes de formar soluções viscosas. Alguns deles também formam géis em meio aquoso, mesmo em baixas concentrações e, em alguns casos, esses polímeros são utilizados como substratos para desenvolvimento de novos produtos, por ex., após uma derivatização química ${ }^{2}$. A produção de exopolissacarídeos (EPS) microbianos não se encontra exposta às alterações climáticas, à contaminação marinha ou à problemas nas colheitas, que prejudicam a oferta e alteram o custo de produção das gomas tradicionais ${ }^{3}$. São, ainda, menos susceptíveis à variabilidade em suas propriedades químicas e físicas, mantendo o padrão de qualidade, pois sua produção pode ser controlada cuidadosamente; além disso, sua recuperação e purificação apresentam dificuldades menores, comparadas aos vegetais, como também há técnicas de genética molecular que permitem obter polissacarídeos microbianos com propriedades específicas, as quais ainda não são possíveis ${ }^{4,5}$ nos vegetais.

Vários EPS microbianos já são aceitos como produtos biotecnológicos e, embora apresentem muitas vantagens, o custo de produção para a maioria deles ainda é relativamente alto quando comparado àqueles obtidos de vegetais e algas marinhas, devido aos processos de produção, intensivos investimentos em capital e energia. Assim, existem poucos microrganismos que produzem EPS com rendimentos satisfatórios para desenvolvimento em escala industrial e, conseqüentemente, sua exploração comercial ainda é baixa. Entretanto, para alguns polímeros, a relação custo/benefício justifica a produção, por possuírem propriedades químicas e físicas únicas ou superiores às dos polissacarídeos tradicionais ${ }^{3,6}$, como por ex., a alta viscosidade, o maior poder geleificante, a compatibilidade com uma grande variedade de sais em ampla faixa de

*e-mail: corradi@prudente.unesp.br
pH e temperatura, a estabilidade em elevadas concentrações iônicas, a alta solubilidade em água e, também, a ação sinérgica com outros polissacarídeos ${ }^{7}$.

Todas estas propriedades são diretamente dependentes das características químicas, físicas e estruturais de cada polímero, como massa molecular (número de unidades por molécula), configuração anomérica ( $\alpha$ ou $\beta$ ), conformação (linear, ramificada, helicoidal, agregação entre cadeias), presença de grupamentos (carboxila, entre outros), tipo e grau de substituição dos monômeros, posição das ligações glicosídicas entre os resíduos, entre outras. Desta forma, a caracterização química dos polímeros é necessária para o direcionamento de suas aplicações industriais ${ }^{8-10}$.

Os polissacarídeos fúngicos, tanto pertencentes à parede celular como os extracelulares, têm sido investigados por apresentarem uma variedade de respostas biológicas de defesa, tais como atividade antitumoral, antiinflamatória e imuno-moduladora ${ }^{11}$. A aplicação terapêutica parece depender da estrutura química e da conformação espacial de cada macromolécula, sendo que pequenas diferenças estruturais de cada polímero resultam em características peculiares para novas aplicações biotecnológicas ${ }^{12}$.

\section{POLISSACARÍDEOS E EXOPOLISSACARÍDEOS}

Polissacarídeos são macromoléculas naturais encontradas em todos os organismos vivos, constituindo um grupo de compostos dos mais abundantes e importantes da biosfera como, por ex., celulose e amido nas plantas ${ }^{13}$ e glicogênio nos animais.

Estes polímeros são constituídos de unidades monossacarídicas unidas por ligações glicosídicas diferindo entre si na unidade e no grau de ramificação destas, no tipo de ligações que as unem e no comprimento de suas cadeias, apresentando diferentes composições e funções, sendo classificados como homopolímeros ou heteropolímeros ${ }^{8}$. Os homopolissacarídeos possuem um único tipo de unidade monomérica, como ex. amido e glicogênio, os quais servem como forma de armazenamento de glucose, e celulose e quitina que atuam como elementos estruturais das paredes das células vegetais e de exoesqueletos de animais, respectivamente. Os heteropolissacarídeos contêm duas ou mais unidades monoméricas 
diferentes, como os peptidoglicanos, que fazem parte da camada rígida da parede celular das células bacterianas e fornecem um suporte extracelular para os organismos de todos os reinos naturais. O ácido hialurônico constitui um outro exemplo que confere resistência e flexibilidade às cartilagens e tendões. Existem polissacarídeos associados a proteínas, os proteoglicanos, que possuem propriedades lubrificantes e promovem alta viscosidade a algumas secreções extracelulares e glicoproteínas, que correspondem à proteína conjugada a oligossacarídeos ou polissacarídeos de baixa massa molecular ${ }^{14}$.

Os microrganismos (algas, bactérias e fungos), assim como as plantas, produzem diferentes polissacarídeos com diversas e importantes aplicações biotecnológicas. As algas produzem o ágar, usado como agente geleificante. Esses polissacarídeos têm sido usados para modificar a viscosidade de fluidos, estabilizar suspensões, flocular partículas, encapsular materiais e produzir emulsões ${ }^{6}$. São, ainda, empregados como agentes de troca iônica, filtros moleculares e agentes de recuperação de óleos ${ }^{13}$.

Os exopolissacarídeos são definidos como polissacarídeos extracelulares, produzidos por alguns fungos e bactérias ${ }^{15}$. Dependendo do sistema microbiano, alguns exopolissacarídeos formam cápsulas ao redor da célula tornando-se parte da parede celular, enquanto outros formam limos fora da parede celular e têm habilidade para se difundirem dentro da fase líquida durante o processo de fermentação. Como resultado da produção do exopolissacarídeo, a viscosidade e a reologia da fermentação podem produzir mudanças no meio de cultivo, começando como um fluido Newtoniano de baixa viscosidade e finalizando como um fluido não-Newtoniano de elevada viscosidade. Os microrganismos que produzem grandes quantidades de limo têm maior potencial para comercialização, considerando ser um processo mais simples ${ }^{16}$ a recuperação dos exopolissacarídeos do meio de fermentação.

No ambiente natural em que os microrganismos são encontrados, tais polímeros podem estar associados à virulência, como no caso de plantas ou patógenos animais, à interação planta-microrganismo, à proteção da célula microbiana contra dessecação ou, ainda, ao ataque por bacteriófagos e protozoários ${ }^{6}$.

A maioria dos polissacarídeos produzidos responde diretamente aos fatores ambientais. Para alguns microrganismos, a fonte de carbono determina a quantidade de polissacarídeo formado, bem como a qualidade do produto sintetizado ${ }^{15}$.

A manipulação da composição do EPS por modificação no meio de cultivo é um mecanismo possível pelo qual as propriedades do polímero podem ser alteradas para aplicação final. Trabalhos experimentais sobre este assunto ainda são limitados, mas indicam que, embora não seja possível alterar a estrutura ou a composição da unidade básica repetitiva do polissacarídeo, é possível mudar o grau de substituição da unidade repetitiva por vários grupos, bem como o grau de polimerização ${ }^{16,17}$.

\section{FUNGOS PRODUTORES DE GLUCANAS}

Fungos são organismos heterotróficos (decompositores de matéria orgânica ou parasitas) que crescem rapidamente e formam filamentos celulares microscópicos denominados hifas, cujo conjunto constitui uma espécie de tecido próprio dos fungos, o micélio, responsável por todas as funções vegetativas do organismo ${ }^{18}$. A obtenção de alimento efetua-se por absorção através das paredes das células, sendo que os elementos nutritivos devem estar na forma de solução. O micélio segrega enzimas especiais que atuam sobre as substâncias, liqüefazendo-as, como é o caso dos fungos ligninolíticos. Em outras situações, o micélio emite órgãos chama- dos haustórios, que penetram no tecido dos organismos hospedeiros, absorvendo o alimento ${ }^{19}$.

Com base em critérios quimiotaxonômicos, estes microrganismos são classificados como oomicetos, zigomicetos, basidiomicetos, ascomicetos e deuteromicetos, incluindo espécies parasitas, saprófitas e simbiontes. Algumas espécies de fungos são bastante conhecidas por causarem doenças nas plantas; ${ }^{5}$ entretanto, por vezes, as substâncias que produzem podem ter aplicações médicas. É o caso dos fungos do gênero Penicillium, que são produtores de antibióticos; da cravagem do centeio, Claviceps purpurea, que contém vários alcalóides, entre eles ergonovina e ergotamina, que atuam como vasoconstritore ${ }^{20}$ e dos produtores de $\beta$-glucanas, Botryosphaeria rhodina, Sclerotium glucanicum, entre vários outros, com ampla aplicação comercial e farmacêutica ${ }^{5}$.

Os polissacarídeos constituem uma importante percentagem da biomassa fúngica; a parede da hifa, por ex., contém mais de 75\% deste tipo de biomolécula. Além de atuarem como elemento de suporte para as hifas, alguns polissacarídeos constituem uma capa extracelular ao redor do micélio, proporcionando um suporte para adesão das enzimas excretadas e participando na degradação da lignina, como uma fonte indireta de peróxido de hidrogênio ${ }^{1}$. As glucanas também podem contribuir para manter o $\mathrm{pH}$ ótimo para as enzimas ligninolíticas, além de impedirem a desidratação das hifas e de regularem a concentração de glucose extracelular. Essas moléculas ficam parcialmente dissolvidas no meio de cultivo quando o fungo cresce em meio líquido ${ }^{21}$.

O fungo ligninolítico mais estudado, Phanerochaete chrysosporium, geralmente degrada apenas lignina em culturas na fase estacionária, formando uma capa polissacarídica. Entretanto, alguns mutantes degradam lignina quando cultivados em meio líquido e, diferentemente da linhagem selvagem, produzem o exopolissacarídeo sob estas condições. A adição de uma glucanase específica ao cultivo interrompe a degradação da lignina, sugerindo que o EPS produzido pelo fungo pode desempenhar um papel importante no processo de biodegradação da macromolécula ${ }^{21}$.

A produção de EPS por fungos do gênero Pleurotus tem sido descrita em diferentes condições de cultivo ${ }^{22}$. O fungo Phlebia radiata, um degradador ativo da madeira, produz quantidades significativas de lacases e peroxidases ligninolíticas e, também, um polissacarídeo extracelular cuja estrutura química foi determinada por Krcmar e colaboradores ${ }^{23}$.

Outros fungos produtores de glucanas extracelulares, também denominadas de exoglucanas, são mostrados na Tabela 1 com suas respectivas ligações glicosídicas.

\section{CARACTERIZAÇÃO QUÍMICA DAS GLUCANAS FÚNGICAS}

Vários são os registros na literatura sobre interpretações estruturais diferentes de um mesmo exopolissacarídeo produzido por um microrganismo. Segundo Seviour e colaboradores ${ }^{3}$ a explicação para essa variação estrutural seria a produção de mais de um exopolissacarídeo pelo mesmo microrganismo. Essa suposição parece correta, principalmente, pelo fato de um número muito pequeno de polímeros ter sido previamente purificado, levando, na maior parte das vezes, à determinação errônea da estrutura. Portanto, para investigar as propriedades e reações dessas moléculas é necessário primeiro isolá-las e purificá-las até a homogeneidade.

Segundo Pazur ${ }^{8}$ os procedimentos de purificação devem ser efetuados até que a composição da macromolécula em estudo se mantenha constante por dois métodos diferentes. O comportamento das glucanas na filtração em gel ou em troca-iônica propicia informações acerca da pureza da preparação polissacarídica. Nes- 
Tabela 1. Fungos produtores de glucanas e suas respectivas ligações glicosídicas

\begin{tabular}{|c|c|}
\hline Microrganismos & Ligação Glicosídica \\
\hline Alternaria solani ${ }^{24}$ & $\beta(1 \rightarrow 3)$ \\
\hline Amanita muscaria ${ }^{25}$ & $\alpha(1 \rightarrow 3)$ \\
\hline Aspergillus nidulans ${ }^{26}$ & $\alpha(1 \rightarrow 3)$ \\
\hline Aureobasidium pullulans ${ }^{27-29}$ & $\alpha(1 \rightarrow 4 ; 1 \rightarrow 6)$ \\
\hline Botryosphaeria rhodina ${ }^{7}$ & $\beta(1 \rightarrow 3 ; 1 \rightarrow 6)$ \\
\hline Botryosphaeria sp. ${ }^{30}$ & $\beta(1 \rightarrow 3 ; 1 \rightarrow 6)$ \\
\hline Botrytis cinerea ${ }^{31}$ & $\beta(1 \rightarrow 3 ; 1 \rightarrow 6)$ \\
\hline Claviceps purpurea ${ }^{32}$ & $\beta(1 \rightarrow 3)$ \\
\hline Epicoccum nigrum $^{28}$ & $\beta(1 \rightarrow 3 ; 1 \rightarrow 6)$ \\
\hline Microdochium nivale ${ }^{33}$ & $\beta(1 \rightarrow 4)$ \\
\hline Paecylomycees lilacinus ${ }^{34}$ & $\beta(1 \rightarrow 3)$ \\
\hline Pestalotia $s p .{ }^{35}$ & $\beta(1 \rightarrow 3 ; 1 \rightarrow 6)$ \\
\hline Phanerochaete chrysosporium ${ }^{36}$ & $\beta(1 \rightarrow 3 ; 1 \rightarrow 6)$ \\
\hline Phytophthora parasitica ${ }^{37}$ & $\beta(1 \rightarrow 3 ; 1 \rightarrow 6)$ \\
\hline Phlebia radiata ${ }^{23}$ & $\beta(1 \rightarrow 3 ; 1 \rightarrow 6)$ \\
\hline Phoma herbarum ${ }^{7}$ & $\beta(1 \rightarrow 3 ; 1 \rightarrow 6)$ \\
\hline Pleurotus tuber-regium ${ }^{38}$ & $\beta(1 \rightarrow 3 ; 1 \rightarrow 6)$ \\
\hline Poria $\operatorname{cocos}^{39}$ & $\beta(1 \rightarrow 3)$ e $\alpha(1 \rightarrow 3)$ \\
\hline Schizophyllum commune $e^{40}$ & $\beta(1 \rightarrow 3 ; 1 \rightarrow 6)$ \\
\hline Sclerotium glucanicum ${ }^{33,41}$ & $\beta(1 \rightarrow 3 ; 1 \rightarrow 6)$ \\
\hline Tremella menseterica ${ }^{3}$ & $\alpha(1 \rightarrow 4 ; 1 \rightarrow 6)$ \\
\hline
\end{tabular}

se procedimento, a preparação é passada através de uma coluna de gel ou troca-iônica e os eluatos são analisados para carboidratos. A simetria no pico de eluição é indicativo de homogeneidade ${ }^{30}$.

A primeira medida a ser tomada a respeito da análise estrutural de um polissacarídeo é conhecer os resíduos monossacarídicos que o constituem ${ }^{14}$. Os polímeros possuem velocidades de hidrólise diferentes, que variam de acordo com o tamanho do anel hemiacetálico, a conformação dos monossacarídeos, configuração anomérica e natureza das ligações glicosídicas, presença de grupos funcionais na molécula, intensidade das interações intra e intermoleculares e outras ${ }^{20,21}$. Para obtenção de condições ideais para hidrólise parcial ou total, é necessário efetuar-se curvas de hidrólise, através das quais são estabelecidos fatores como concentração do ácido na solução, temperatura e tempo. Esses cuidados asseguram que um maior número de ligações glicosídicas sejam rompidas, evitando a degradação dos monossacarídeos liberados pela hidrólise, a partir do polissacarídeo. A análise do hidrolisado é geralmente efetuada por cromatografia e a seleção do método a ser usado depende do equipamento disponível no laboratório ${ }^{1}$.

Para determinar a posição da ligação glicosídica, um dos métodos mais utilizados é a metilação ${ }^{8}$. Esta técnica pode fornecer uma indicação do grau de polimerização do polissacarídeo, a partir da determinação quantitativa dos éteres metílicos de monossacarídeos ${ }^{9}$. Há vários métodos de metilação de açúcares, que se baseiam nos trabalhos pioneiros elaborados por Denham e Woodhouse e por Haworth ${ }^{42}$. O procedimento padrão usado por esses autores consistia no tratamento do polímero com $\mathrm{NaOH}$ e sulfato de dimetila. Embora não exista um método satisfatório para se obter uma metilação completa de todos os grupos hidroxilas livres de carbonos vicinais, o método de Hakomori é considerado eficaz na metilação de glucanas neutras solúveis em dimetilsulfóxido. Resultados satisfatórios são, geralmente, alcançados pela execução do experimento por duas ou três vezes ${ }^{8,42}$. Com a introdução de microtécnicas para metilação e subseqüente análi- se dos produtos de hidrólise por cromatografia líquida - gasosa e espectrometria de massa, a utilidade do método tem sido ainda mais valorizada.

A degradação de Smith, outra técnica útil na determinação da estrutura dos polímeros, consiste na redução com boroidreto de sódio dos grupos aldeídicos formados na oxidação do polissacarídeo com o periodato, seguida da hidrólise ácida total e/ou parcial. Os produtos resultantes desta degradação formam a mistura de polióis e glicoaldeídos, provenientes das unidades oxidadas pelo periodato, e de açúcares livres, quando o polissacarídeo contém unidades resistentes à oxidação ${ }^{8,14}$. Esses resultados confirmam aqueles obtidos pela metilação.

Embora a metilação seja considerada um dos principais métodos para estudar a estrutura dos carboidratos, não é capaz de proporcionar informação adequada a respeito da configuração anomérica e do arranjo espacial das várias unidades monossacarídicas. A ressonância magnética de núcleo tem sido utilizada, com sucesso, na determinação da estrutura. Esta técnica baseia-se na observação de que núcleos magnéticos tais como ${ }^{1} \mathrm{H},{ }^{13} \mathrm{C},{ }^{31} \mathrm{P}$ e ${ }^{15} \mathrm{~N}$ podem absorver energia em freqüências características, quando colocados em um campo magnético forte. A frequiência de ressonância de um núcleo particular, expressa como deslocamento químico $(\delta)$, é sensível ao ambiente químico da molécula, tornando o RMN uma técnica valiosíssima para estudos estruturais ${ }^{43,44}$. Cada molécula tem um espectro de RMN característico que pode ser usado como uma impressão digital ("fingerprint") da mesma. Uma outra grande vantagem desta técnica é seu caráter não destrutivo, tornando-a uma das primeiras técnicas escolhidas para caracterização estrutural.

Outra análise espectroscópica também utilizada para estudo de glucanas é o infravermelho que, mesmo fornecendo espectros complexos, é usualmente aplicado à identificação de características estruturais específicas, como a configuração das ligações glicosídicas, a presença de ácido urônico, através do sinal característico da carboxila e, até mesmo, a presença de proteína ${ }^{21,23}$.

Algumas glucanas, para terem suas estruturas completamente elucidadas, necessitam de reações adicionais tais como acetólise, que é um método complementar da hidrólise ácida ${ }^{37}$. Em geral, os fragmentos de oligossacarídeos obtidos na acetólise de um polissacarídeo contendo diferentes tipos de ligações glicosídicas são diferentes dos obtidos na hidrólise ácida parcial deste mesmo polímero. Isto porque ligações glicosídicas mais vulneráveis na acetólise são muitas vezes mais resistentes à hidrólise ácida, como por ex. a ligação $1 \rightarrow 6$.

Geralmente, diante desse conjunto de análises realizado com todo critério científico, é possível determinar a estrutura química dos exopolissacarídeos.

\section{DESCRIÇÃO DAS PRINCIPAIS GLUCANAS FÚNGICAS}

Glucanas são polímeros de glucose amplamente distribuídos na natureza e classificadas conforme o tipo de ligação glicosídica $[\alpha, \beta]$ da cadeia principal. Nos fungos, estas moléculas participam como componentes menores do citossol, da parede celular e como polissacarídeos excretados ao meio ${ }^{45}$.

São poucos os registros sobre $\alpha$-glucanas fúngicas na literatura. Um exemplo é aquela produzida pelo Tremella mesenterica, que contém ligações do tipo $\alpha-(1 \rightarrow 4)$ e $(1 \rightarrow 6)$ na proporção de $2: 1$ e é estruturalmente muito semelhante à pululana (Figura 1-I), uma $\alpha$-glucana produzida pelo fungo Aureobasidium pullulans, mais estudada até o momento ${ }^{3,29}$. Com base nos resultados da análise por infravermelho e oxidação pelo periodato, sugeriu-se que a pululana seja uma $\alpha$-glucana constituída de subunidades repetitivas 

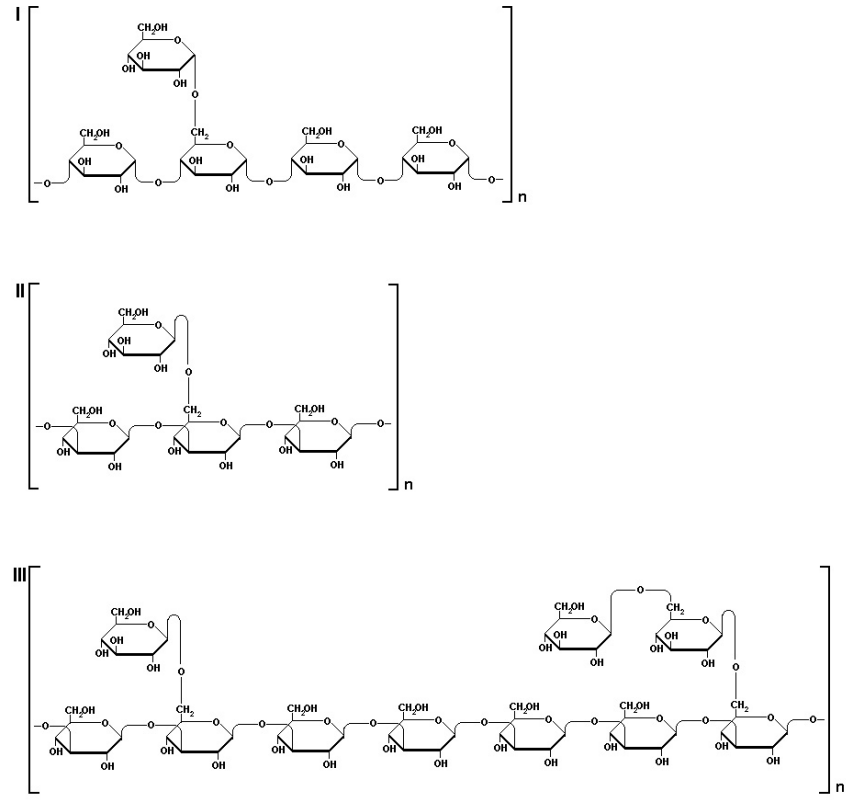

Figura 1. Fórmulas estruturais das glucanas. I. Pululana $[\alpha-(1 \rightarrow 4)(1 \rightarrow 6)-$ glucana]; II. escleroglucana $[\beta-(1 \rightarrow 3)(1 \rightarrow 6)$-glucana], substituída por unidades glucosídicas com freqüência variada; III. botriosferana [ $\beta$ $(1 \rightarrow 3)(1 \rightarrow 6)$-glucana], substituída por unidades glucosídicas elou gentiobiosídicas a cada cinco unidades da cadeia principal

de maltotriose e um pequeno número de unidades de maltotetraose unidas por ligações $(1 \rightarrow 6)$. Alguns autores acreditam que a proporção de ligações $\alpha-(1 \rightarrow 4)$ e $(1 \rightarrow 6)$ possa variar em função da linhagem fúngica estudada, embora tais variações nem sempre ocorram após purificação. A pululana não é o único polissacarídeo produzido por este fungo $\mathrm{o}^{46}$, porém é o de maior interesse industrial (com cerca de mais de 150 patentes para sua produção e uso), apesar dos problemas encontrados durante a produção, como a elevada viscosidade do meio de cultivo, presença de melanina e ocorrência de pululanólise ${ }^{29,47}$.

Zhang e colaboradores ${ }^{2}$ extraíram uma glucana do corpo de frutificação do basidiomiceto Ganoderma lucidum. Após resultados das análises de metilação, oxidação pelo periodato, ressonância magnética nuclear de carbono 13 e espectroscopia de infravermelho, a molécula foi caracterizada como um polímero linear constituído de resíduos glucosídicos $\alpha-(1 \rightarrow 3)$ ligados. Derivados sulfatados desta molécula têm apresentado atividade antitumor contra o carcinoma de Ehrlich.

As $\beta$-glucanas são a forma predominante encontrada em fun$\operatorname{gos}^{45}$. Na parede celular dos fungos, estes polímeros estão normalmente ligados à proteína, lipídeos e outros carboidratos, tais como manana. A função específica da glucana na fisiologia do fungo não está completamente esclarecida; entretanto, considera-se que sua principal função estrutural seja auxiliar na manutenção da rigidez e integridade da parede celular ${ }^{3}$. Tem sido sugerido que as glucanas extracelulares dos fungos ligninolíticos desempenham um importante papel na degradação de lignina, como fonte indireta de peróxido de hidrogênio ${ }^{23}$. Outra participação deste exopolissacarídeo no metabolismo do fungo, em particular daqueles que degradam a madeira, seria a de atuar como um suporte para adsorção das enzimas excretadas e, finalmente, acredita-se que o exopolissacarídeo protegeria a hifa da desidratação, bem como regularia a concentração de glucose extracelular ${ }^{48}$.

A escleroglucana (Figura 1-II), pertencente ao grupo das $\beta$ glucanas, é produzida por várias espécies de fungos do gênero
Sclerotium $^{3,16}$. A cadeia principal é composta por unidades de glucose $\beta$ - $(1 \rightarrow 3)$, às quais as unidades glucosídicas $\beta$ - $(1 \rightarrow 6)$ podem estar ligadas regular ou randomicamente, com frequiências diferentes $^{1,5}$. Grandes variações no grau de ramificação podem ser observadas, o que afeta a solubilidade da molécula ${ }^{6}$. Na escleroglucana derivada de Schizophyllum commune, posteriormente denominada esquizofilana (massa molecular entre $1,3 \times 10^{5}$ a $6,0 \mathrm{x}$ $10^{6} \mathrm{Da}$ ), as ramificações estão distribuídas regularmente a cada três unidades da cadeia principal. A escleroglucana obtida do Sclerotium glucanicum tem menor massa molecular, aproximadamente $18 \mathrm{x}$ $10^{3} \mathrm{Da}$, com substituintes glucopiranosídicos a cada cinco ou seis unidades da cadeia principal e, neste caso, é um polissacarídeo solúvel que gera soluções altamente viscosas com as moléculas poliméricas, adaptando-se à conformação em tripla-hélice ${ }^{6}$.

O fungo Pestalotia sp. 815, quando cultivado em meio contendo glucose, produz um exopolissacarídeo constituído por unidades glucosídicas. Esta glucana, designada de pestalotana, apresentou $[\alpha]_{\mathrm{D}}^{25}-0,1^{\circ}$ (c $\left.0,5, \mathrm{NaOH} 1 \mathrm{M}\right)$ e massa molecular aproximada de 2 x $10^{6} \mathrm{Da}$. Estudos químicos e enzimáticos indicaram que a pestalotana tem estrutura muito ramificada, contendo uma cadeia principal constituída de resíduos glucopiranosídicos $\beta-(1 \rightarrow 3)$ ligados e sustituídos em C-6 por glucose, em três de cada cinco unidades da cadeia principal e, em menor proporção, por resíduos gentiobiosídicos ${ }^{35}$.

Uma glucana insolúvel em água (G-A), com rotação óptica específica de $+12,8^{\circ}$ (c. $0,4, \mathrm{NaOH} 1 \mathrm{M}$ ), foi obtida a partir do extrato alcalino do corpo de frutificação do fungo Ganoderma japonicum. A G-A mostrou-se homogênea nas análises de filtração em gel, eletroforese e ultracentrifugação. A massa molecular e o grau de polimerização foram, respectivamente, estimados em $82 \mathrm{x}$ $10^{3} \mathrm{Da}$ e 330 . As análises de espectroscopia de infravermelho e ressonância magnética nuclear de próton, juntamente com os resultados da oxidação por trióxido de cromo, indicaram que as ligações glicosídicas apresentavam a configuração $\beta$. A partir dos resultados encontrados nas análises de metilação, oxidação pelo periodato, degradação de Smith e hidrólise ácida parcial, os autores concluíram que a G-A é composta de uma cadeia principal constituída por unidades glucopiranosídicas $\beta-(1 \rightarrow 3)$ ligadas e com substituição em C-6 por grupos $\beta$-D-glucopiranosídicos possuindo, em média, uma substituição a cada 30 resíduos da cadeia principal $^{49}$.

Vários polissacarídeos têm sido obtidos a partir do extrato aquoso do corpo de frutificação do fungo Ganoderma lucidum $^{50}$. Uma das glucanas isoladas foi caracterizada como tendo uma cadeia principal constituída de unidades glucopiranosídicas $\beta-(1 \rightarrow 3)$ substituídas em O-6 por uma unidade de glucose $\mathrm{e}^{2,51}$. Glucanas com estrutura química semelhantes também foram isoladas dos esporos deste mesmo fungo. Mais recentemente, Bao e colaboradores ${ }^{52}$ purificaram e caracterizaram uma $\beta$-D-glucana complexa constituída de uma cadeia principal $(1 \rightarrow 6)$ substituída em C-3 e C-4 por unidades glucosídicas na forma de mono-, di- e trissacarídeos. A estrutura química desta molécula foi determinada após experimentos de caracterização da glucana nativa e de seus fragmentos oligossacarídicos obtidos por hidrólise e acetólise parciais.

Sone e colaboradores (apud ref. 53) isolaram $\beta$-D-glucanas solúveis e insolúveis em água, além de dois heteropolissacarídeos a partir do corpo de frutificação do fungo Auricularia auricula-judae. Foi demonstrado, através de técnicas de metilação e degradação de Smith, que a glucana solúvel em água consistia de uma cadeia principal de unidades glucopiranosídicas $\beta-(1 \rightarrow 3)$ substituídas em C-6 por unidades $\beta$-D-glucopiranosídicas, em uma proporção aproximada de 30\%. A $\beta$-D-glucana, insolúvel em água, apresentou estrutura semelhante, mas com maior grau de ramificação. 
Um polissacarídeo solúvel em álcali, obtido do corpo de frutificação de Pleurotus tuber-regium eluiu como um único pico por cromatografia de filtração em gel, indicando homogeneidade e apresentou massa molecular de 4,3 x $10^{5} \mathrm{Da}$. Os resultados obtidos por hidrólise ácida total, oxidação pelo periodato, degradação de Smith, metilação, infravermelho e RMN ${ }^{13} \mathrm{C}$ indicaram tratar-se de uma glucana com cadeia principal constituída de resíduos glucopiranosídicos $\beta$ - $(1 \rightarrow 3)$ substituídos em C-6, a cada três unidades da cadeia principal, por uma unidade de glucose ${ }^{38}$.

Um polissacarídeo fúngico extracelular, denominado epiglucana, sintetizado por três diferentes linhagens do Epicoccum nigrum foi caracterizado como uma $\beta$-D-glucana $(1 \rightarrow 3,1 \rightarrow 6)$. Análises de metilação, $\mathrm{RMN}{ }^{13} \mathrm{C}$ e digestão enzimática mostraram leves variações na frequência das ramificações entre os polímeros produzidos pelas três linhagens examinadas. A cadeia principal $\beta$ $(1 \rightarrow 3)$ apresentou ramificações em C-6 numa freqüência maior que as glucanas homólogas, escleroglucana e esquizofilana, encontradas nos fungos Sclerotium spp e Schizophyllum commune, respectivamente ${ }^{28}$.

Polissacarídeos extracelulares produzidos por cultivo submerso a partir de diferentes espécies de Pleurotus apresentaram composição e estrutura semelhantes ${ }^{21}$. A principal fração, que correspondia a mais de $80 \%$ de todos os polissacarídeos, foi investigada em detalhes. A baixa solubilidade da molécula foi atribuída à elevada massa molecular e para sua caracterização, um procedimento especial de dissolução e diálise foi elaborado. A análise de metilação mostrou tratar-se de uma $\beta$-D-glucana $(1 \rightarrow 3)$ com aproximadamente $25 \%$ das unidades ramificadas em C-6. Estes resultados foram confirmados pela intensidade dos sinais de C-3 e C-6, livre e substituído, no espectro de $\mathrm{RMN}{ }^{13} \mathrm{C}$. Estudos adicionais de degradação de Smith e acetólise indicaram que as cadeias laterais ligadas em C-6 eram constituídas de uma unidade de glucose ligada a cada duas ou três unidades da cadeia principal ${ }^{21}$.

A análise do material extracelular produzido pelo fungo Phlebia radiata revelou a presença de glucose $(62 \%)$, galactose $(16 \%)$, manose $(13 \%)$, xilose $(4 \%)$ e fucose $(5 \%)$, juntamente com proteína. A purificação deste material pela tripsina rendeu um exopolissacarídeo rico em glucose. Análises de metilação e RMN ${ }^{13} \mathrm{C}$ identificaram o EPS como uma $\beta$-D-glucana $(1 \rightarrow 3)$ altamente ramificada em $\mathrm{C}-6^{23}$.

Uma exoglucana insolúvel em água foi isolada do meio de cultivo do fungo Microdochium nivale. O espectro na região do infravermelho apresentou um pico em $890 \mathrm{~cm}^{-1}$, indicando a configuração $\beta$ do polímero. Os resultados dos experimentos de metilação mostraram tratar-se de um polímero linear constituído por unidades de glucose $1 \rightarrow 4$ ligadas. Este resultado foi confirmado pela digestão do polissacarídeo com celulase, uma vez que somente glucose foi liberada. Este foi o primeiro registro de um fungo produtor de celulose como um polissacarídeo extracelular ${ }^{33}$.

O fungo filamentoso Phoma herbarum foi isolado do solo da Antártica continental e avaliado como produtor de EPS. Os resultados foram positivos e a maior quantidade de EPS (13,6 g/L) foi alcançada quando sorbitol foi utilizado como fonte de carbono e $\mathrm{NaNO}_{3}$ como fonte de nitrogênio. O polissacarídeo foi purificado por precipitação em etanol e filtração em gel e caracterizado como um homopolímero de glucose com massa molecular de 7,4 x $10^{6}$ $\mathrm{Da}$; a análise estrutural efetuada por metilação e degradação enzimática ( $\beta-1,3$ glucosidase) indicou a presença de ligações $\beta$ $(1 \rightarrow 3)$ e $\beta(1 \rightarrow 6)^{7}$.

Vários polissacarídeos foram isolados da parede celular do Phytophthora parasitica, um fungo fitopatogênico. A mistura polissacarídica foi fracionada por cromatografias sucessivas em DEAE-celulose, Sephadex G-25, Concanavalina-A Sepharose e
Sephadex G-200. Os polissacarídeos neutros consistiam de uma $\beta$ D-glucana $(1 \rightarrow 3,1 \rightarrow 6)$ cuja massa molecular ficou entre $9 \times 10^{3}$ a $200 \times 10^{3} \mathrm{Da}$. Todos estes polissacarídeos eram constituídos por uma cadeia principal de resíduos de glucose $\beta-(1 \rightarrow 3)$ ligados. Eles diferiam pela presença das cadeias laterais consistindo de D-glucose e D-laminaribiose para os polímeros de menor massa molar, e laminaritriose para aqueles de maior massa molar ${ }^{37}$.

Um polissacarídeo insolúvel em água foi obtido do extrato alcalino do corpo de frutificação do fungo Amanita muscaria. Por cromatografia de filtração em gel em Toyopearl HW-65F o material foi eluído como um pico simétrico e sua massa molecular estimada em cerca de $42 \times 10^{3}$ Da. A análise por cromatografia gasosa dos derivados acetilados, provenientes da hidrólise ácida, mostrou principalmente glucose, com pequenas quantidades de manose. $\mathrm{O}$ polissacarídeo foi completamente metilado e os resultados mostraram apenas dois derivados identificados como 2,4,6-tri-O-metilD-glucose e traços de 2,3,4,6-tetra-O-metil-D-glucose. O sinal encontrado em 99,62 ppm no espectro de $\mathrm{RMN}{ }^{13} \mathrm{C}$ foi atribuído aos átomos do carbono anomérico de unidades $\alpha$-D-glucopiranosídicas $(1 \rightarrow 3)$ substituídas ${ }^{25}$.

Bes e colaboradores ${ }^{36}$ detectaram no meio de cultivo do Phanerochaete chrysosporium um polissacarídeo que, após separação da massa micelial por centrifugação, diálise e precipitação em etanol, se mostrou solúvel em água. Estudos químicos e espectroscópicos confirmaram ser um polímero constituído por uma cadeia principal de unidades de glucose $\beta-(1 \rightarrow 3)$ substituídas em C-6 por unidades glucopiranosídicas.

Uma glucana microparticulada insolúvel em água foi isolada da parede celular do fungo Saccharomyces cerevisiae. Por cromatografia líquida de exclusão molecular o polímero apresentou massa molecular média de 3,53 x $10^{6} \mathrm{Da}$, polidispersividade de 1,12 , viscosidade intrínsica de $0,366 \mathrm{dL} / \mathrm{g}$ e, aproximadamente, 196 unidades de glucose por cadeia. A espectroscopia de RMN ${ }^{13} \mathrm{C}$ da glucana mostrou seis sinais em 102,60 (C-1), 85,98 (C-3), 76,14 (C-5), 72,59 (C-2), 68,23 (C-4) e 60,71 (C-6) ppm. Estes deslocamentos concordam com aqueles previamente registrados para outras $\beta$-glucanas $(1 \rightarrow 3)$ e os assinalamentos foram confirmados por RMN 2D pelos espectros COSY e HETCOR ${ }^{54,55}$.

Osaku e colaboradores ${ }^{56}$ monitoraram a produção de exopolissacarídeos a partir do fungo Thelephora terrestris. Os exopolissacarídeos foram preparados por precipitação em etanol e fracionados em dois componentes usando-se cromatografia em coluna de DEAE-Sepharose. A fração neutra, refracionada em Sepharose CL-6B, resultou em três picos denominados de NeP1, $\mathrm{NeP} 2$ e NeP3. A fração NeP1 mostrou ser um heteropolímero, enquanto NeP2 e NeP3 foram caracterizadas como uma glucana $\beta$ $(1 \rightarrow 6)$ semelhante à pustulana e uma glucana $\beta-(1 \rightarrow 3)$, respectivamente.

Recentemente, foram realizados estudos dos efeitos do meio de cultivo nas características químicas e físicas dos exopolissacarídeos produzidos pelo micélio de Poria cocos, obtendo-se cinco frações polissacarídicas. As características químicas e físicas foram determinadas por espectroscopia de infravermelho, RMN ${ }^{13} \mathrm{C}$, cromatografia gasosa dos derivados metilados, determinação da massa molar por cromatografia de exclusão molecular e estudos viscosimétricos. Três frações foram caracterizadas como heteropolímeros compostos, principalmente, por $\alpha$-D-glucose, manose e galactose. As outras frações eram compostas exclusivamente por glucose, sendo uma constituída por $\alpha$-D-glucana $(1 \rightarrow 3)$ e a outra por $\beta$-D-glucana $(1 \rightarrow 3)$, mas ambas lineares ${ }^{39}$.

Um exopolissacarídeo, denominado botriosferana (Figura 1III), produzido pelo fungo ascomiceto Botryosphaeria sp, foi recentemente caracterizado. Esse metabólito foi obtido por precipi- 
tação etanólica, purificado por cromatografia de filtração em gel e submetido à hidrólise ácida para determinação de seus constituintes monossacarídicos. Os resultados das análises por cromatografia de troca-iônica de alta pressão revelaram $98 \%$ de glucose. Os espectros de infravermelho e $\mathrm{RMN}{ }^{13} \mathrm{C}$ mostraram que todas as ligações glicosídicas apresentavam configuração $\beta$. Os resultados das análises de metilação e degradação de Smith indicaram que a botriosferana é uma glucana $\beta$-D- $(1 \rightarrow 3)$ com cerca de $22 \%$ de ramificações em C-6. Após hidrólise ácida parcial, foi demonstrado que as ramificações consistem de resíduos glucopiranosídicos e gentiobiosídicos unidos à cadeia principal por ligações glicosídicas $\beta$-D- $(1 \rightarrow 6)$, presentes a cada cinco resíduos da cadeia principal ${ }^{30}$.

\section{APLICAÇÃO DAS GLUCANAS FÚNGICAS}

Vários exopolissacarídeos produzidos por fungos ainda não foram adequadamente explorados e poucos têm sido produzidos em escala industrial. Muitos destes biopolímeros apresentam propriedades reológicas que são valiosas para emprego industrial. Entre os polissacarídeos fúngicos com aplicação comercial podem ser citados o escleroglucana e a pululana ${ }^{1,13}$.

Os EPS microbianos são mais conhecidos por suas propriedades espessantes, geleificantes e emulsificantes; porém, outra possibilidade de aplicação destes biopolímeros é na saúde humana. Várias pesquisas sobre polissacarídeos fúngicos têm se concentrado em aplicá-los como agentes antitumorai ${ }^{57,58}$. Os polissacarídeos que apresentam esta atividade são glucanas intimamente relacionadas à estrutura da escleroglucana ${ }^{59}$. Alguns polissacarídeos ricos em L-fucose também podem apresentar aplicação na área médica, como por ex., impedindo a colonização do pulmão por células tumorais (efeito anticancerígeno). Podem ainda ser utilizados no controle da formação de leucócitos (efeito antiinflamatório), no tratamento da artrite reumatóide, na síntese de antígenos para produção de anticorpos (imunização), no efeito protetor contra danos oxidativos no $\mathrm{DNA}^{60}$, na interação com lipoproteínas de baixa densidade por meio de forças de van der Waals, que resulta na eliminação da fração lipídica do compartimento sanguíneo ${ }^{47,61}$ e em cosméticos como agentes de hidratação da pele ${ }^{62}$.

A pululana, uma $\alpha$-glucana produzida pelo fungo Aureobasidium pullulans, é resistente ao óleo e daí sua aplicação em poços de petróleo. Este EPS forma filmes solúveis em água com baixa permeabilidade ao oxigênio, sendo utilizado para revestir alimentos pois retém o sabor e a aparência. Soluções deste EPS podem também ser usadas para formar coberturas, sem odor e sabor, sobre materiais alimentícios. A aplicação da pululana e de seus derivados tem sido proposta e patenteada, principalmente pelos japoneses que a comparam aos polímeros sintéticos de álcool polivinílico nas propriedades de transparência, brilho, maciez e elasticidade ${ }^{15}$. A pululana é um bom adesivo e pode ser usada na preparação de algumas fibras, como um componente de sistemas aquosos bifásicos, como padrões de massa molecular de baixa dispersão para calibrar HPLC. Uma nova particularidade do uso da pululana é como pré-biótico para promover seletivamente o crescimento de Bifidobacterium spp no intestino humano, seguindo sua incorporação sobre alimentos de dieta especializada ${ }^{1,6}$.

Entre os polissacarídeos biologicamente ativos, as D-glucanas tipo $\beta$ - $(1 \rightarrow 3)$ e $\beta$ - $(1 \rightarrow 3 ; 1 \rightarrow 6)$ revelaram-se compostos potentes, sendo efetivos contra tumores autólogos tanto quanto alogenêicos e singenêicos. Lentinana e esquizofilana, duas glucanas fúngicas $\beta-(1 \rightarrow 3 ; 1 \rightarrow 6)$, tornaram-se clinicamente relevantes como imunoadjuvantes na terapia contra o câncer, no Japão. Estas glucanas, não citotóxicas, supostamente expressam seu efeito antitumor por estimulação do sistema imunológico do hospedeiro.
Acredita-se que a ação aconteça, principalmente, pela ativação das células T "helper", células natural "killer" e macrófagos citotóxicos tanto quanto por um aumento nas células T citotóxicas ${ }^{63-65}$. Estudos recentes mostram que as glucanas $\beta-(1 \rightarrow 3)$ modulam o sistema imunológico pela ativação dos macrófagos através da ligação do polímero a receptores específicos destas células ${ }^{45,66}$. Esta atividade antitumor parece estar relacionada à estrutura química da cadeia principal da glucana na forma de hélice tríplice, à frequiência e complexidade das cadeias laterais e à sua massa molecular ${ }^{28}$. Portanto, decifrar as características estruturais destes polímeros é um pré-requisito essencial para entender como as glucanas fúngicas são reconhecidas pelo sistema imune (77-72 $^{\text {. }}$

A atividade antitumoral das soluções de pestalotana, do poliol de pestalotana (preparado por degradação de Smith da glucana nativa) e do poliol submetido a tratamento ultra-sônico para obtenção das moléculas despolimerizadas foi testada em tumores alogenêicos e singenêicos (Sarcoma 180 e carcinoma de Erlich) em linhagens apropriadas de camundongos. Em todas as situações estudadas verificou-se que a melhor atividade estava relacionada ao poliol de pestalotana desagregado por ultra-som, que foi obtido de solução menos viscosa ${ }^{35}$. Uma glucana semelhante obtida de Cordyceps ophioglossoides (CO-1) inibiu fortemente o desenvolvimento do tumor Sarcoma 180; o poliol derivado de CO-1, também preparado por degradação de Smith, mostrou-se ainda mais potente na mesma dosagem, provavelmente devido à maior solubilidade da molécula em água ${ }^{73}$.

O maior obstáculo da utilização clínica das $\beta$-glucanas é a falta relativa de solubilidade em meio aquoso. Segundo Williams e colaboradores $^{74}$, $\beta$-D-glucanas $(1 \rightarrow 3)$ isoladas da parede celular da levedura Saccharomyces cerevisiae como micropartículas insolúveis (1-2 $\mu \mathrm{m})$ não induzem nenhuma toxicidade quando administradas intralesão ou topicamente, porém o uso sistêmico (intravenoso) da forma microparticulada está associado com hepatoesplenomegalia, formação de granulomas, microembolia e aumento da sensibilidade às endotoxinas. Um dos primeiros procedimentos empregados para solubilização destas moléculas microparticuladas foi a sulfatação da glucana de Saccharomyces cerevisiae. O objetivo primário foi aumentar a polaridade da molécula aumentando, conseqüentemente, sua solubilidade em água e preservando a ação imunobiológica. Este objetivo foi alcançado, considerando-se que as glucanas sulfatadas exerceram potente atividade antimicrobiana e antineoplásica e, particularmente, um efeito inibitório ${ }^{74}$ contra o vírus causador da imunodeficência humana (HIV).

O corpo de frutificação de Ganoderma lucidum, denominado "Lingzhi" na China e "Rheishi" no Japão, tem sido usado tradicionalmente na medicina chinesa e em outros países asiáticos ${ }^{75}$. Seus efeitos médicos contra câncer, hipertensão, hepatite e hipercolesterolemia foram demonstrados por estudos farmacológicos nas últimas duas ou três décadas. Este fungo tem atraído considerável atenção, particularmente, devido aos polissacarídeos isolados do corpo de frutificação, micélio e esporos ${ }^{50,52}$, por apresentarem atividade antitumoral e hipoglicêmica ${ }^{51}$. A modificação destas glucanas por reações de sulfatação gerou derivados com ação antiviral $^{2}$, cujo mecanismo de ação, embora não completamente esclarecido, acredita-se ser proveniente de uma interação entre o polissacarídeo carregado negativamente, pela presença dos grupos sulfatos, com a porção de uma proteína do envelope viral, carregada positivamente.

Um produto solúvel em água obtido pela carboximetilação de uma glucana $\beta-(1 \rightarrow 3)$, linear, extraída do fungo Amanita muscaria mostrou potente atividade antitumoral contra Sarcoma 180, enquanto uma outra glucana obtida de forma semelhante deixou de apre- 
sentar atividade quando extensivamente carboximetilada ${ }^{25}$.

Sasaki e Takasuka (apud ref. 2) relataram que a lentinana, uma glucana $\beta-(1 \rightarrow 3)$ com ramificação em C-6, somente apresentou atividade antitumoral após ter sido hidrolisada parcialmente para obtenção de um polímero menor (1,6 x 10 $\left.10^{4} \mathrm{Da}\right)$, enquanto a esquizofilana e grifolana apresentaram atividade antitumoral ${ }^{76}$ apenas com massas moleculares superiores a $1 \times 10^{4} \mathrm{Da}$.

Portanto, verifica-se que a massa molecular, a conformação e a modificação química dos polissacarídeos afetam as ações antitumoral e imunomodulatória ${ }^{28}$. Assim, um entendimento básico da estrutura secundária do polissacarídeo é essencial para uma interpretação bem sucedida da atividade biológica.

Geralmente, tem sido aceito que glucanas $\beta$-D- $(1 \rightarrow 3)$ com estrutura ordenada constituída por uma cadeia flexível e com conformação em hélice tripla têm elevada atividade antitumoral ${ }^{76}$. Entretanto, existem alguns polissacarídeos com estruturas químicas diferentes que apresentam esta mesma atividade, tais como hetero $\beta$ glucanas, heteroglucanas, $\alpha$-glucanas e $\alpha$-glucana-proteínas ${ }^{77}$.

A descoberta de que moléculas purificadas, estruturalmente semelhantes quando analisadas por $\mathrm{RMN}{ }^{13} \mathrm{C}$ e metilação, apresentavam ação antitumoral diferente levou os autores a suspeitarem da possibilidade de classes específicas da massa molecular. Para verificar esta hipótese, o estudo da polidispersividade por cromatografia de exclusão molecular, bem como a viscosidade das soluções passaram a ser analisados ${ }^{78-80}$, abrindo-se campo para a investigação físico-química dos polissacarídeos e mais uma referência para compreensão dos resultados da atividade biológica.

\section{CONSIDERAÇÕES GERAIS}

De acordo com os dados relatados nesta revisão, observou-se que a purificação e caracterização química das diversas glucanas têm evidenciado propriedades peculiares, responsáveis pelo seu potencial de aplicação industrial.

Embora a aplicação das $\beta$-glucanas como agente antitumoral depare-se com a baixa solubilidade destas moléculas em água, limitando a eficiência farmacológica, estão sendo realizados estudos sobre modificações químicas, o que se acredita, levará a bons resultados para estas e novas aplicações. Ainda, de acordo com Giese e colaboradores ${ }^{81}$, o desenvolvimento de pesquisas sobre a obtenção de polissacaridases irá vencer as barreiras determinadas pela insolubilidade dos polímeros, gerando moléculas de pequena massa molecular, solúveis, e que preservem a atividade biológica.

\section{AGRADECIMENTOS}

À FAPESP, CNPq e PROPPG-UEL-PR pelo apoio financeiro.

\section{REFERÊNCIAS}

1. Barbosa, A. M.; Cunha, P. D. T.; Pigatto, M. M.; Corradi da Silva, M. L.; Semina 2004, 25, 29.

2. Zhang, L.; Zhang, M.; Zhou, Q.; Chen, J.; Zeng, F.; Biosci., Biotechnol., Biochem. 2000, 64, 2172.

3. Seviour, R. J.; Stasinopoulos, S. J.; Auer, D. P. F.; Gibbs, P. A.; Crit. Rev. Biotechnol. 1992, 12, 279.

4. Shah, V.; Ray, A.; Garg, N.; Madamwar, D.; Curr. Microbiol. 2000, 40, 274.

5. Selbmann, L.; Crognale, S.; Petruccioli, M.; Lett. Appl. Microbiol. 2002, 34,51 .

6. Sutherland, I. W.; Tibtech January 1998, 16, 41

7. Selbmann, L.; Onofri, S.; Fenice, M.; Federice, F.; Petruccioli, M.; Res. Microbiol. 2002, 153, 585.

8. Pazur, J. H. Em Carbohydrate Analysis: A Practical Approach; Chaplin, M. F.; Kennedy, J. F., eds.; Oxford University Press; Oxford, 1994, p. 73.
9. Lindberg, B.; Methods Enzymol. 1972, 28, 178.

10. Macedo, M. G.; Lacroix, C.; Gardner, N. J.; Champagne, C. P.; Int. Dairy J. 2002, 12, 419 .

11. Wasser, S. P.; Appl. Microbiol. Biotechnol. 2002, 60, 258.

12. Colleoni-Sirghie, M.; Fulton, D. B.; White, P. J.; Carbohydr. Polym. 2003, 54, 237

13. Glazer, A.; Nikaido, H.; Microbial Biotechnology: fundaments of applied microbiology, W.H. Freeman: New York, 1995, p. 50.

14. Nelson, D. L.; Cox, M. M.; Lehninger Principles of Biochemistry, $3^{\text {rd }}$ ed., Worth Publishers: New York, 2000, cap. 9.

15. Kang, K. S; Cottrell, I. W. Em Polysaccharides in Microbial Technology, Peppler, H. J.; Perlman, D., eds.; Academic Press: New York, 1979, p. 417.

16. Margaritis, A.; Pace, G. W. Em Comprehensive Biotechnology: the principles, applications and regulations of biotechnology in industry, agriculture and medicine; Blanch, H. W.; Drew, S.; Wang, D. I. C., eds.; Pergamon Press: New York, 1985, p. 1005.

17. Cottrell, I. W.; Kang, K. S.; Kovacs, P. Em Handbook of water-soluble gums and resins; Davidson, R.; ed.; Mac Graw Hill: New York, 1980, p. 13.

18. Hudson, H. J. Em Fungal Biology; Willis, A. J.; Sleigh, M. A.; eds.; Edward Arnold Ltd: London, 1986, p. 289.

19. Griffin, D. H.; Fungal Physiology; $2^{\text {nd }}$ ed.; Wiley-Lis: New York, 1994, p. 168.

20. Bonatti, S.; Monografia de Graduação, Universidade Estadual Paulista Júlio de Mesquita Filho, Brasil, 2002.

21. Gutiérrez, A.; Prieto, A.; Martinez, A. T.; Carbohydr. Res. 1996, $281,143$.

22. Burns, P. J.; Ueo, P.; Keshavarz, T.; Roller, S.; Evans, C. S.; Enzyme Microb. Technol. 1994, 16, 566.

23. Krcmar, P.; Novotny, C.; Maraias, M-F; Joseleau, J-P.; Int. J. Biol. Macromol. 1999, 24, 61 .

24. Goatley, J. L.; Can. J. Microbiol. 1968, 14, 1063.

25. Kiho, T.; Yoshida, I.; Katsuragawa, M.; Sakushima, M.; Usui, S.; Ukai, S.; Biol. Pharm. Bull. 1994, 17, 1460.

26. Leal, J. A.; Ruperez, P.; Trans. Br. Mycol. Soc. 1978, 70, 115.

27. Gibbs, P. A.; Serviour, R. J.; Appl. Microbiol. Biotechnol. 1998, 49, 168.

28. Schmid, F.; Stone, B. A.; Cdougall, B. M.; Bacic, A.; Martin, K. L.; Brownlee, R. T. C.; Chai, E.; Seviour, R. J.; Carbohydr. Res. 2001, 331, 163.

29. Kachhawa, D.; Bhattacharjee, P.; Singhal, R.; Carbohydr. Polym. 2003, 52, 25.

30. Barbosa, A. M.; Steluti, R. M.; Dekker, R. F. H.; Cardoso, M. S.; Corradi da Silva, M. L.; Carbohydr. Res. 2003, 338, 1691.

31. Dubourdieu, D.; Ribereau-Gayon, P.; Fournet, B.; Carbohydr. Res. 1981, 93, 294.

32. Buck, K. W.; Chen, A. W.; Dickerson, A. G.; Chain, E. B.; J. Gen. Appl. Microbiol. 1968, 51, 337.

33. Schweiger-Hufnagel, U.; Ono, T.; Izumi, K.; Hufnagel, P.; Morita, N.; Kaga, H.; Morita, M.; Hoshino, T.; Yumoto, I.; Matsumoto, N.; Yoshida, M.; Sawada, M. T.; Okuyama, H.; Biotechnol. Lett. 2000, 22, 183.

34. Sarkar, J. M.; Hennebert, G. L.; Mayaudon, J.; Biotechnol. Lett. 1986, 8, 769.

35. Misaki, A.; Kawaguchi, K.; Miyaji, H.; Nagae, H.; Hokkoku, S.; Kakuta, A.; Sasaki, T.; Carbohydr. Res. 1984, 129, 209.

36. Bes, B.; Pettersson, B.; Lennholm, H.; Iversen, T.; Eriksson, K-E.; Biotechnol. Appl. Biochem. 1987, 9, 310.

37. Fabre, I.; Bruneteau, M.; Ricci, P.; Michel, G.; Eur. J. Biochem. 1984, 142, 99.

38. Chenghua, D.; Xiangliang, Y.; Xiaoman, G.; Yan, W.; Jingyan, Z.; Huibi, X.; Carbohydr. Res. 2000, 328, 629.

39. Jin, Y.; Zhang, L.; Chen, L.; Chen, Y.; Cheung, P. C. K.; Chen, L.; Carbohydr. Res. 2003, 338, 1507.

40. Tabata, K.; Wataru, I.; Kojima, T.; Kawabata, S.; Misaki, A.; Carbohydr. Res. 1981, 89, 121.

41. Johnson, J.; Kirkwood, W.; Isaki, A.; Nelson, T. E.; Scaletti, J.V.; Smith, F.; Chem. Ind. 1963, 41, 820.

42. Haworth, W. N.; J. Chem. Soc. 1915, 107, 8.

43. Gorin, P. A. J. Em Advances in Carbohydrate Chemistry and Biochemistry; Whistler, R. L.; Be Miller, J. N., eds.; Academic Press: New York, 1981, vol. 38 , p. 12.

44. Gorin, P. A. J.; Barreto-Bergter, E.; The Polysaccharides 1983, 2, 365.

45. Williams, D. L.; Mediators Inflamm. 1997, 6, 247.

46. Navarini, L.; Bella, J.; Flabani, A.; Gilli, R.; Riza, V.; Int. J. Biol. Macromol. 1996, 19, 157.

47. Shingel, I. K.; Carbohydr. Res. 2004, 339, 447.

48. Buchala, A. J.; Leisola, M.; Carbohydr. Res. 1987, 165, 146.

49. Ukai, S.; Yokoyama, S.; Hara, C.; Kiho, T.; Carbohydr. Res. 1982, 105, 237.

50. Bao, X-F.; Wang, X-S.; Dong, Q.; Fang, J-N.; Li, X-Y.; Phytochemistry 2002, 59, 175. 
51. Bao, X-F.; Zhen, Y.; Ruan, L.; Fang, J-N.; Chem. Pharm. Bull. 2002, 50, 623.

52. Bao, X.; Fang, J.; Li, X.; Biosci. Biotechnol. Biochem. 2001, 65, 2384.

53. Zhang, L.; Yang, L.; Ding, Q.; Chen, X.; Carbohydr. Res. 1995, 270, 1.

54. Ensley, H.; Tobias, B.; Pretus, H.; Mcnamee, R. B.; Jones, E. L.; Browder, I. W.; Williams, D. L.; Carbohydr. Res. 1994, 258, 307.

55. Müller, A.; Rice, P. J.; Ensley, H. E.; Coogan, P. S.; Kalbfleisch, J. H.; Kelley, J. L.; Love, E. J.; Portera, C. A.; Ha, T.; Browder, I. W.; Williams, D. L.; J. Immunol. 1996, 156, 3418.

56. Osaku, C. A.; Sassaki, G. L.; Zancan, G. T.; Iacomini, M.; FEMS Microbiol. Lett. 2002, 216, 145.

57. Maziero, R.; Cavazzoni, V.; Bononi, V. L. R.; Rev. Microbiol. 1999, 30, 77.

58. Hwang, H-J, Kim, S-W, Choi, J-W, Yun, J-W.; Enzyme Microb. Technol. 2003, 33, 309.

59. Yang, F-C.; Liau, C-B.; Process Biochem. 1998, 33, 547.

60. Slamenová, D.; Lábaj, J.; Krizková, L.; Kogan, Gsandula, J.; Bresgen, N.; Eckl, P.; Cancer Lett. 2003, 198, 153.

61. Engstad, C. S.; Engstad, R. E.; Olsen, J-O.; Osterud, B.; Int. Immunopharm. 2002, 2, 1585

62. Vanhooren, P. T.; Vandame, E. J. J.; Chem. Technol. Biotechnol. 1999, 74, 479.

63. Gomaa, K.; Kraus, J.;Rokopf, F.; Roper, H.; Franz, G. J.; Cancer Res. Clin. Oncol. 1992, 118, 136

64. Vetvicka, V.; Thornton, B. P.; Ross, G. D.; J. Clin. Invest. 1996, 98, 50.

65. Cheung, N-K. V.; Modak, S.; Vickers, A.; Knuckles, B.; Cancer Immunol. Imunother. 2002, 51, 557.

66. Young, S. H.; Ye, J.; Frazer, D. G.; Shi, X.; Castranova, V.; J. Biol. Chem. 2001, 276, 20781.
67. Kishida, E.; Sone, Y.; Misaki, A.; Carbohydr Polym. 1992, 17, 89.

68. Bohn, J. A.; Bemiller, J. N.; Carbohydr. Polym. 1995, 28, 3.

69. Kulicke, W-M.; Lettau, A. I.; Thielking, H.; Carbohydr. Res. 1997, 297, 135.

70. Lowman, D. W.; Feerguson, D. A.; Williams, D. L.; Carbohydr. Res. 2003, 338, 1491.

71. Vetvicka, V.; Yvin, J-C.; Int. Immunopharmacol. 2004, 4, 721.

72. Ishibashi, K-I; Miura, N. N.; Adachi, Y.; Tamura, H.; Tanaka, S.; Ohno, N.; FEMS Immmunol. Med. Microbiol. 2004, 42, 155.

73. Yamada, H.; Kawaguchi, N.; Ohmori, T.; Takeshita, Y.; Taneya, S-I.; Miyazaki, T.; Carbohydr. Res. 1984, 125, 107.

74. Williams, D. L.; Pretus, H. A.; Mcnamee, R. B.; Jones, E. L.; Ensley, H. E.; Browder, I. W.; Carbohydr. Res. 1992, 235, 247.

75. Wang, Y-Y.; Khoo, K-H.; Chen, S-T.; Lin, C-C.; Wong, C-H.; Lin, C-H.; Bioorg. Med. Chem. 2002, 10, 1057.

76. Borchers, A. T.; Stern, J. S.; Hackman, R. M.; Keen, C. L.; Keen, C. L.; Gershwin, M.E.; Pharm. Soc. Exp. Biol. Med. 1999, 221, 281.

77. Mizuno, M.; Morimoto, M.; Minato, K.; Tsuchida, H.; Biosci. Biotechnol. Biochem. 1998, 62, 434

78. Villain-Simonnet, A.; Milas, M.; Rinaudo, M.; Int. J. Biological Macromol. 2000, 27, 65 .

79. Calazans, G. M. T.; Lima, R. C.; França, F. P.; Lopes, C. E.; Int. J. Biol. Macromol. 2000, 27, 245 .

80. Lazaridou, A.; Biliarderis, C. G.; Izydorczyk, M. S.; Food Hydrocolloids. 2003, 17, 693

81. Giese, E. C.; Barbosa, A. M.; Corradi da Silva, M. L.; Rev. Biotechnol. C. Desenvol. 2003, 30, 97. 